\title{
Optical near-field study of photonic crystal tapers
}

\author{
Delphine Néel, ${ }^{1,4}$ Taha Benyattou, ${ }^{1, *}$ Pablo Sanchis, ${ }^{2}$ Javier Martí, ${ }^{2}$ \\ Wim Bogaerts, ${ }^{3}$ Pieter Dumon, ${ }^{3}$ and Roel Baets ${ }^{3}$ \\ ${ }^{1}$ Institut des Nanotechnologies de Lyon (INL), CNRS, UMR 5270, Université de Lyon, \\ INSA-Lyon, Bât "Blaise Pascal," 7 avenue Jean Capelle, \\ Villeurbanne F-69621, France \\ ${ }^{2}$ Nanophotonics Technology Center, Universidad Politécnica de Valencia, 46022 Valencia, Spain \\ ${ }^{3}$ IMEC, Department of Information Technology,Ghent University, B-9000 Ghent, Belgium \\ ${ }^{4}$ Currently with the Institut d'Electronique Fondamentale, Centre Scientifique d'Orsay, \\ 91405 Orsay Cedex, France \\ ${ }^{*}$ Corresponding author: taha.benyattou@ insa-lyon.fr
}

Received 5 June 2009; revised 6 October 2009; accepted 6 October 2009; posted 6 October 2009 (Doc. ID 111907); published 20 October 2009

\begin{abstract}
A scanning near-field optical microscopy study is undertaken on photonic crystal tapers. These tapers are used to couple a $3 \mu$ mide multimode dielectric waveguide to a single-line defect monomode photonic crystal waveguide. Two kinds of taper, with or without a localized defect, are compared. Higher transmission efficiency is obtained when a defect is utilized. The near-field study at $1550 \mathrm{~nm}$ shows experimentally that this defect prevents leaky resonant states to appear in the taper and thus permits a decrease in out-of-plane losses. These observations are supported by band diagram calculations. (C2009 Optical Society of America OCIS codes: $\quad 130.5296,180.4243,250.5300,160.5298,230.0250$.
\end{abstract}

\section{Introduction}

Photonic crystals (PCs) are interesting structures for the development of highly integrated optical circuits $[1,2]$. In this context, the coupling between conventional dielectric waveguides and planar PC waveguides is an important issue. Among all the existing coupling techniques [3-5], PC tapers appear as an interesting approach to couple large dielectric waveguides and planar PC waveguides. These types of taper present the advantages of a small coupling length and a high coupling efficiency achieved over a large frequency range [6]. We report the near-field optical study of a photonic crystal taper. Scanning near-field optical microscopy (SNOM) is crucial for good comprehension of the coupling process as it allows local mapping of the electromagnetic field of the taper structure under working conditions, with a resolution less than the wavelength.

0003-6935/09/305718-04\$15.00/0

(C) 2009 Optical Society of America

\section{Experimental Conditions}

An efficient coupling technique between dielectric and planar photonic crystal waveguides was previously demonstrated in [7]. In this technique, a localized defect is introduced in a PC taper linking a large dielectric waveguide to a $\mathrm{PC}$ waveguide. The studied structure is composed of a $3 \mu \mathrm{m}$ wide multimode dielectric waveguide coupled to a $0.6 \mathrm{~W}$ single-mode photonic crystal waveguide through a taper (Fig. 1) with $W=753 \mathrm{~nm}$ being the width of a single-line missing-hole defect waveguide. The photonic crystal is composed of a triangular lattice of airholes. The lattice period is $a=435 \mathrm{~nm}$ and the hole radius is $R=0.264 a$. A localized defect of radius $R$ is inserted into the input taper at a distance of $3.9 a$ from the first column of holes that forms the PC taper. A similar defect is set in the output taper. The holes are etched in a silicon-on-insulator (SOI) wafer composed of a $220 \mathrm{~nm}$ thick silicon layer, stuck on a $1 \mu \mathrm{m}$ silica layer, deposited on a bare silicon substrate. Only the silicon layer is etched. Patterns were fabricated by deep-UV lithography at $248 \mathrm{~nm}$, 


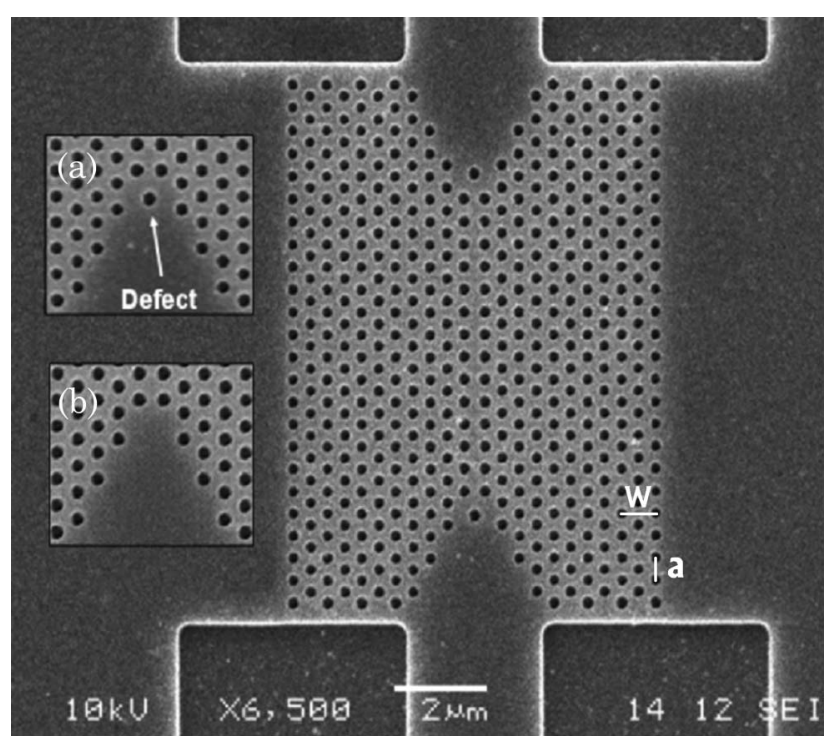

Fig. 1. SEM image of the structure with $W$ as the width of a $W 1$ waveguide and $a$ as the lattice period. The inset shows a detailed view of the taper (a) with a defect and (b) without a defect.

followed by inductive coupled plasma etching with a chemistry based on $\mathrm{Cl}_{2} / \mathrm{HBr} / \mathrm{He} / \mathrm{O}_{2}$ [8] .

Transmission measurements in $\overline{\mathrm{T}} \mathrm{E}$ polarization (electric field $E$ lies within the silicon layer plane) showed that transmission efficiency up to $60 \%$ was achieved at $1550 \mathrm{~nm}$ for the structure with defects, whereas it was only approximately $20 \%$ without defects (see [7]). Moreover, between 1450 and $1570 \mathrm{~nm}$ the taper with defects transmits 3 to 4 times more signal than the taper without defects.

A SNOM study is undertaken to correlate this increase with the taper structure. For these experiments, we used a commercial SNOM (Omicron, Taunusstein, Germany) that operates in the collection mode. Light from a tunable laser is injected by the facet of the sample through a monomode IR cleaved fiber. The electromagnetic field on the surface of the waveguide is collected by a nonmetallized SNOM tip and then recorded by an InGaAs photodetector. The SNOM tip is a tapered optical fiber obtained by chemical attack in hydrofluorhydric acid $[9,10]$, whose apex radius is approximately $100 \mathrm{~nm}$.

\section{Experimental Results}

SNOM images were achieved on structures without [Fig. 2(a)] and with [Fig. 2(b)] localized defects in TE polarization at $1550 \mathrm{~nm}$. It can be noted that optical images are slightly asymmetric because of the difficulty to achieve a perfectly symmetric injection in the multimode $3 \mu \mathrm{m}$ wide waveguide. On both images, the guided light can clearly be seen in the $0.6 \mathrm{~W}$ waveguide. This guided wave has the expected periodicity for a Bloch wave, that is, $430 \mathrm{~nm}$, which matches the lattice period [11]. A standing wave is also present in the waveguide because of the reflections that occur at both ends, leading to a FabryPerot effect. The modulation of the standing wave (a)

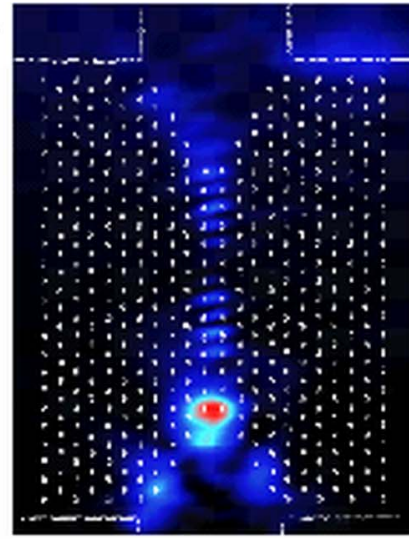

(b)

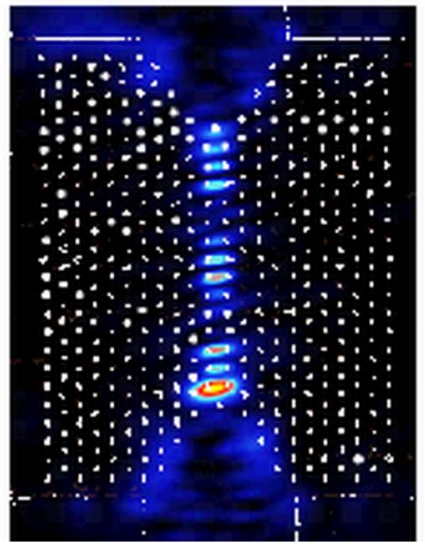

6550 (a. u.)

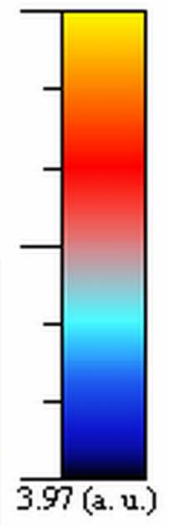

Fig. 2. (Color online) SNOM images recorded at $1550 \mathrm{~nm}$ : (a) without localized defects and (b) with localized defects. The position of the holes deduced from the topographic images is reported on the optical images. The unit of the signal intensity is arbitrary. The size of each image is $9 \mu \mathrm{m} \times 12 \mu \mathrm{m}$. Light moves from bottom to top.

is superimposed on the modulation of the Bloch wave.

The important point with regard to the SNOM images is the presence of a hot spot at the entrance of the $0.6 \mathrm{~W}$ waveguide for the structure without defects. Using far-field measurements, we checked that this hot spot is related to out-of-plane scattering. A zoomed view of the input taper is presented in Fig. 3(a). For clarity, we sliced the taper structure into one-period section waveguides, which are labeled as $0.6,2.6$, and $4.6 \mathrm{~W}$ with respect to their corresponding width. Note that the scattered light originates mainly from the $2.6 \mathrm{~W}$ region that connects the upper part of the taper with the $0.6 \mathrm{~W}$ waveguide. The $2.6 \mathrm{~W}$ section is thus the main source of loss in the taper.

Looking at the taper with a localized defect [Fig. 3(b)], it can be seen that the defect hole is positioned in the strategic region $(2.6 \mathrm{~W})$ where the maximum scattering was previously observed. Here, no signal is detected in the taper region. Only an increase in the Bloch wave signal is observed at the entrance of the $0.6 \mathrm{~W}$ waveguide. For this structure, no scattering was detected in the far field. The evanescent nature of this signal has been checked by achieving experiments at various constant heights. 
(a)

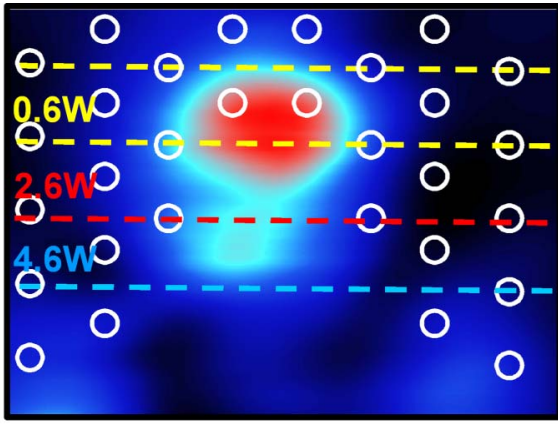

(b)

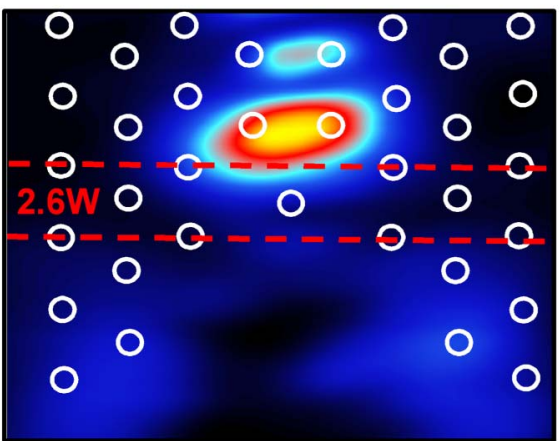

Fig. 3. (Color online) Zoom on SNOM images of the input taper in Fig. 2: (a) without a localized defect and (b) with a localized defect. The position of the holes deduced from the topographic images is reported on the optical images. The unit of the signal intensity is arbitrary. Light moves from bottom to top.

Figure 4 shows the exponential decay of the signal intensity measured in the taper with defects. The curve tends to a constant meaning that there is still a background signal.

\section{Theoretical Analysis}

For a coupling analysis, the $2 \mathrm{D}$ band diagrams of the 0.6 and $2.6 \mathrm{~W}$ sections of the tapers were calculated using the plane wave method [12]. Following the approach developed by Johnson et al. [13], we studied

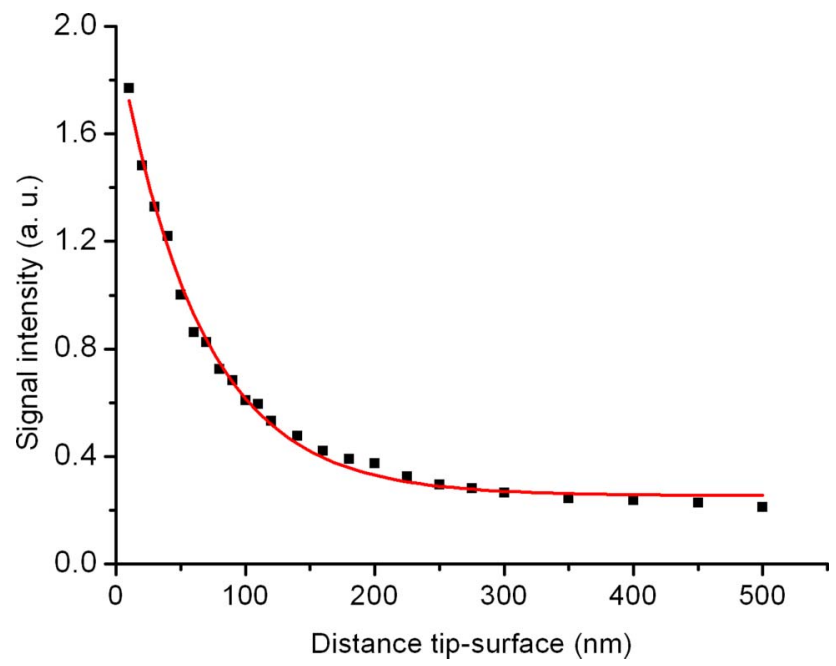

Fig. 4. (Color online) Evolution of the signal intensity depending on the tip-surface distance: squares, experimental points; continuous curve, theoretical curve. the taper structure with a breakdown in slices. In our analysis we assume that the light injected into the dielectric waveguide is even. Consequently, only the even modes are represented on the band diagrams in Fig. 5. Bear in mind that the $0.6 \mathrm{~W}$ waveguide is monomode.

With no localized defect [Fig. 5(a)], only two points are favorable to the coupling. These points correspond to the crossing of the 0.6 and $2.6 \mathrm{~W}$ dispersion curves [see the circles in Fig. 5(a)]. They are located at reduced frequencies of $0 . \overline{286}$ and 0.27 (respectively, $\lambda=1520 \mathrm{~nm}$ and $\lambda=1610 \mathrm{~nm}$ ). Away from these two points, the two modes have different wave vectors that are unfavorable for coupling. Moreover, even for these two points, the slope (i.e., group velocity) of the two modes differs, which leads to important reflections. Thus, these calculations show a large impedance mismatch between the two waveguides. As a consequence, important reflections occur at the entrance of the $0.6 \mathrm{~W}$ waveguide, which give rise to a leaky localized state. This state accounts for a major part of the coupling losses, leading to weak experimental transmission.
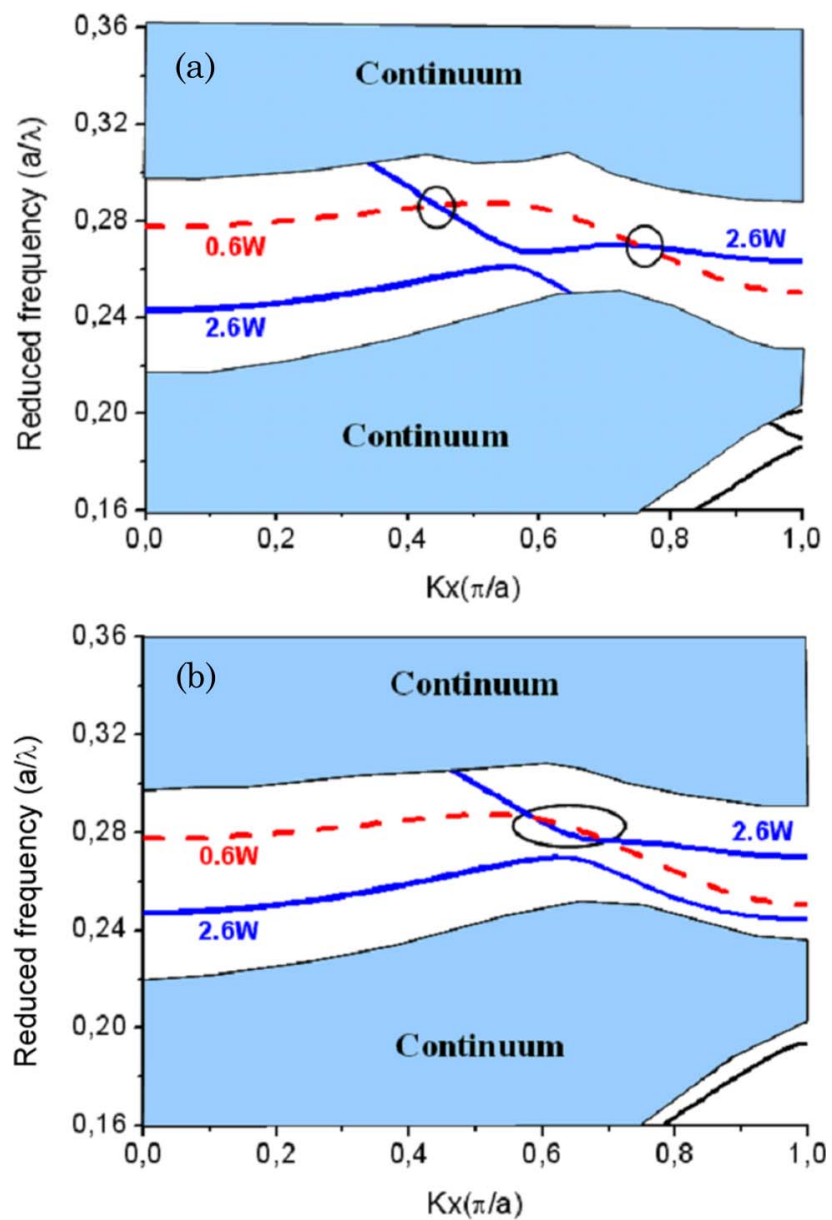

Fig. 5. (Color online) Two-dimensional band diagram of the $2.6 \mathrm{~W}$ waveguide: (a) without a defect and (b) with defects. Only the even modes are represented. The fundamental mode of the $0.6 \mathrm{~W}$ waveguide is represented by dashed curves; the circles correspond to the optimal coupling regions. 
The introduction of a localized defect in the $2.6 \mathrm{~W}$ waveguide shifts the corresponding bands to higher frequencies [Fig. 5(b)]. This can be easily explained if we take into consideration that the hole defect reduces the average index of the guiding region. Therefore, in the frequency range between 0.285 and 0.277 (from 1530 to $1570 \mathrm{~nm}$ ), the $0.6 \mathrm{~W}$ fundamental mode and the refractive $2.6 \mathrm{~W}$ mode merge and their slopes are also close. The coupling efficiency is thus maximal. The cavity effect observed without defects is now broken, which explains the higher transmission efficiency of the taper with a defect.

Following the same procedure, we note that a further improvement of the PC taper would be possible by modifying the $4.6 \mathrm{~W}$ region. We also observed that there is only a slight shift in wavelength between the simulations and the experimental results, which can be assumed to be caused by overexposed or underexposed holes that are due to proximity effects during the lithography process.

\section{Conclusion}

To conclude, the field mapping of PC tapers was performed using scanning near-field optical microscopy. Its unique capability to map the electromagnetic field, with a sublambda resolution in correlation with a topographic view, has been illustrated. Important out-of-plane losses were highlighted for a taper without an optimized defect. The SNOM studies clearly reveal that these losses are related to a leaky state localized at the entrance of the $0.6 \mathrm{~W}$ waveguide. This state is caused by the impedance difference between the different sections of the taper, particularly between the 0.6 and the $2.6 \mathrm{~W}$ sections. The near-field image of the modified taper shows that the presence of the hole defect suppresses the leaky state, leading to a higher coupling efficiency. This behavior was explained using a band diagram analysis of the different sections of the taper. It was shown that the presence of a defect in the taper leads to closer dispersion properties of the 0.6 and $2.6 \mathrm{~W}$ sections.

SNOM images were taken at the microscopy center of Nanoptec in Lyon, France. The SNOM images were screened and treated using the WSxM freeware from Nanotec Electronica.

\section{References}

1. E. Yablonovitch, "Inhibited spontaneous emission in solidstate physics and electronics," Phys. Rev. Lett. 58, 20592062 (1987).

2. J. D. Joannopolous, R. D. Meade, and J. N. Winn, Photonic Crystals: Molding the Flow of Light (Princeton U. Press, 1995).

3. A. Mekis and J. D. Joannopoulos, "Tapered couplers for efficient interfacing between dielectric and photonic crystal waveguides," J. Lightwave Technol. 19, 861-865 (2001).

4. M. E. Potter and R. W. Ziolkowski, "Two compact structures for perpendicular coupling of optical signals between dielectric and photonic crystal waveguides," Opt. Express 10, 691-698 (2002).

5. Y. Xu, R. Lee, and A. Yariv, "Adiabatic coupling between conventional dielectric waveguides and waveguides with discrete translational symmetry," Opt. Lett. 25, 755-757 (2000).

6. M. Dinu, R. L. Willett, K. Baldwin, L. N. Pfeiffer, and K. W. West, "Waveguide tapers and waveguide bends in AlGaAsbased two-dimensional photonic crystals," Appl. Phys. Lett. 83, 4471-4473 (2003).

7. P. Sanchis, J. Marti, W. Bogaerts, P. Dumon, and R. Baets, "Experimental results and 3D analysis of a high efficiency coupling technique for planar photonic crystals," in Proceedings of the IEEE European Conference on Optical Communication (IEEE, 2005), Vol. 2, pp. 181-182.

8. W. Bogaerts, R. Baets, P. Dumon, V. Wiaux, S. Beckx, D. Taillaert, B. Luyssaert, J. Van Campenhout, P. Bienstman, and D. Van Thourhout, "Nanophotonic waveguides in siliconon-insulator fabricated with CMOS technology," J. Lightwave Technol. 23, 401-412 (2005).

9. P. Hoffmann, B. Dutoit, and R. P. Salathé, "Comparison of mechanically drawn and protection layer chemically etched optical fiber tips," Ultramicroscopy 61, 165-170 (1995).

10. D. R. Turner, "Etch procedure for optical fibers," U.S. patent 4,469,554 (4 September 1984).

11. M. J. Martin, T. Benyattou, R. Orobtchouk, A. Talneau, A. Berrier, M. Mulot, and S. Anand, "Evidence of Bloch wave propagation within photonic crystal waveguides," Appl. Phys. B 82, 9-12 (2006).

12. K. M. Leung and Y. F. Liu, "Photon band structures: the planewave method," Phys. Rev. B 41, 10188-10190 (1990).

13. S. G. Johnson, P. Bienstman, M. A. Skorobogatiy, M. Ibanescu, E. Lidorikis, and J. D. Joannopoulos, "Adiabatic theorem and continuous coupled mode theory for efficient taper transition in photonic crystal," Phys. Rev. E 66, 066608 (2002). 